\title{
Is time to scan visual images due to demand characteristics?
}

\author{
PIERRE JOLICOEUR \\ University of Waterloo, Waterloo, Ontario, Canada \\ and \\ STEPHEN M. KOSSLYN \\ Harvard University, Cambridge, Massachusetts
}

\begin{abstract}
The time to scan across an object in a mental image increases with the distance scanned. Does this finding reflect the operation of underlying mechanisms or the effects of demand characteristics? Two types of demand characteristics were considered here. First, the scanning effects were shown to persist even when they conflicted with the experimenters' expectations. Experimenters were led to expect different rates of scanning and different effects of distance on scan times, but the results in all cases showed no hint of experimenter effects, instead displaying the usual increase in time with distance scanned. Second, the scanning effects were shown to persist in the absence of implicit task demands. Effects of distance were observed in an experiment in which subjects were never asked to scan the image; furthermore, such effects were observed only for items that should require spontaneous scanning even though they were randomly intermixed with items that should not, and this distinction was never mentioned nor alluded to in the instructions. Thus, experimenter effects and task demands are not sufficient explanations for the increase in the time to scan across an image as distance is increased.
\end{abstract}

The nature of mental imagery has been hotly debated for years (see Kosslyn, 1980, for a review). The researchers agree that people experience visual images; the disagreement is over how images are actually represented (e.g., see Kosslyn, 1981; Kosslyn \& Pomerantz, 1977; Pylyshyn, 1973, 1981). One position is that image representations are no different in kind from the representations underlying language processing (e.g., Anderson \& Bower, 1973; Pylyshyn, 1973, 1981). Another position is that images are a distinct sort of representation that depicts information by a semantics of resemblance: Each part of an image corresponds to part of an object (as seen from a particular point of view) such that the distances between the parts on the object are preserved by the "distances" between the corresponding parts of the image.

Requests for reprints should be sent either to P. Jolicoeur, Department of Psychology, University of Waterloo, Waterloo, Ontario N2L $3 \mathrm{G}$, or to S. M. Kosslyn, 1236 William James Hall, 33 Kirkland Street, Cambridge, MA 02138. This work was supported by NSF Grant BNS 82-40259 and NIMH Grant MH 39478-01, awarded to Stephen Kosslyn, and by NSERC Grant A2579, awarded to Pierre Jolicoeur. The work was conducted in part while Pierre Jolicoeur was supported by a postgraduate scholarship from the Natural Sciences and Engineering Research Council of Canada and from les Fonds F.C.A.C. pour l'aide et le soutien a la recherce. We wish to thank Tom Carr, Ronald Finke, Margret Intons-Peterson, and Robert Weber for constructive criticism and helpful suggestions, and Sharon Fliegel, Molly Diesing, Ben Bauer, Annette Bauman, Kriss Robinson Stavely, and Dave Rynard for technical assistance, and we again thank our experimenters.
The results of experiments on scanning mental images often have been taken to support the claim that visual images depict information. In these experiments, the typical finding is that the time to scan an imaged object increases linearly with the distance scanned across. The increased time with increasing distance scanned has been taken as evidence that distance is indeed intrinsically represented in mental images (see Kosslyn, 1980; Kosslyn, Ball, \& Reiser, 1978; Pinker, 1980).

The inference that image-scanning times reveal properties of the underlying representation-namely its inherent spatial nature-has been criticized on methodological grounds. One alternative interpretation of the scanning results is that these results reveal nothing other than " $\mathrm{ex}$ perimenter expectancy effects." In this view, subjects (perhaps unconsciously) respond to cues given by the experimenter, leading them to perform as expected: responding more slowly when asked to scan greater distances (see Intons-Peterson, 1983; the "open peer review" of Kosslyn, Pinker, Smith, \& Shwartz, 1979; Mitchell \& Richman, 1980; Richman, Mitchell, \& Reznick, 1979). According to this interpretation, subjects either deduce the purposes and predictions of the experiment from these cues and oblige by responding as expected, or they simply comply with cues about how to behave in the experiment.

The idea that experimenters are somehow leading subjects to behave in a given way in imagery experiments has been investigated by Intons-Peterson (1983). She gave different "predictions" to different experimenters con- 
ducting imagery experiments, and found that the predictions given to the experimenters affected the results. In this paper we focus on the image-scanning paradigm developed by Kosslyn (1973) and Kosslyn et al. (1978), and thus only Intons-Peterson's scanning experiment (Experiment 2) is of immediate relevance. In that experiment, Intons-Peterson required subjects to scan between points on a map that was either physically present or remembered in a mental image. The subjects were asked to image a small dot moving from one point on the map to another point, and to press a button when the dot arrived at its destination. Subjects were tested by experimenters who had been led to hold different expectations about the outcome of the experiment. Some experimenters were led to expect that scanning an imaged map would be faster than scanning a perceived map, whereas other experimenters were led to expect that scanning a perceived map would be faster than scanning an imaged one. The results were as follows (see Figure 3, Intons-Peterson, 1983): (1) In all conditions, response time increased linearly with increases in the distance scanned across; (2) the rate of scanning (i.e., the slope of the increase in scanning time with distance) was the same in all conditions; and, (3) scanning times were generally slower (i.e., the intercept of the function was higher) in the imagery condition when experimenters expected image scanning to be slower than perceptual scanning, but there was no difference in the times when the experimenters believed that perceptual scanning should be slower than image scanning.

Thus, Intons-Peterson's results showed that experimenter expectancies could affect the intercept of the scanning function, but there was no evidence that the slope or the shape of the function could be affected. Given that virtually all theorizing about the mechanisms underlying imagery inferred from mental scanning experiments has focused on the shape and slope of the scanning function, rather than on the intercept, the Intons-Peterson results per se have no direct theoretical implications for theories of image representation. The implications are only indirect-namely they suggest that other more important aspects of the scanning function may also be susceptible to expectancy effects. Experiments 1, 2, and 3 in this paper were designed to discover whether experimenter expectancy effects have caused the characteristic linear increase in response time with distance scanned; if so, then these results indicate nothing about the underlying image representations.

Another alternative interpretation of the scanning results is that they reveal nothing other than subjects' abilities to respond to "task demands." In this view, the instructions implicitly require the subjects to pretend that they are scanning across a real object, and subjects believe (perhaps unconsciously) that longer distances will require more time to scan across (see Pylyshyn, 1981). These beliefs in turn lead the subjects to take more time to respond when longer distances are to be scanned. In contrast to the experimenter expectancy explanation, this interpre- tation does not rest on subjects' being exposed to cues from the experimenter; instead, the claim is that the instructions, by the way they define the task, lead the subjects to produce the expected results. Experiment 4 addresses this issue directly, and it also provides converging evidence against the experimenter expectancy interpretation of image-scanning results.

\section{EXPERIMENT 1}

Given the importance of the actual shape of the relationship between scanning time and distance scanned, we wished to discover whether this relationship was determined by the operation of underlying mechanisms or by experimenter effects. In this experiment, the experimenters were led to believe that a relatively long time would be required to scan between two close locations on an imaged scene because they are "cluttered together." In fact, however, the theory of image scanning presented in Kosslyn (1980) led us to expect generally increasing times to scan longer distances.

\section{Method}

Subjects. Thirty Brandeis University undergraduates volunteered to participate as subjects for pay. No subject participated in more than one condition in any experiment, or in more than one experiment reported in this paper.

Materials. The fictional map used by Kosslyn et al. (1978, Experiment 2) was modified for use in this experiment. The four closest objects were redrawn using heavy black ink, as is illustrated in Figure 1. The experimenters were told that this manipulation should cause these objects to group into a single unit, in accordance with the Gestalt Laws of Proximity and Similarity; these laws were explained to the experimenters, and their purported implications for

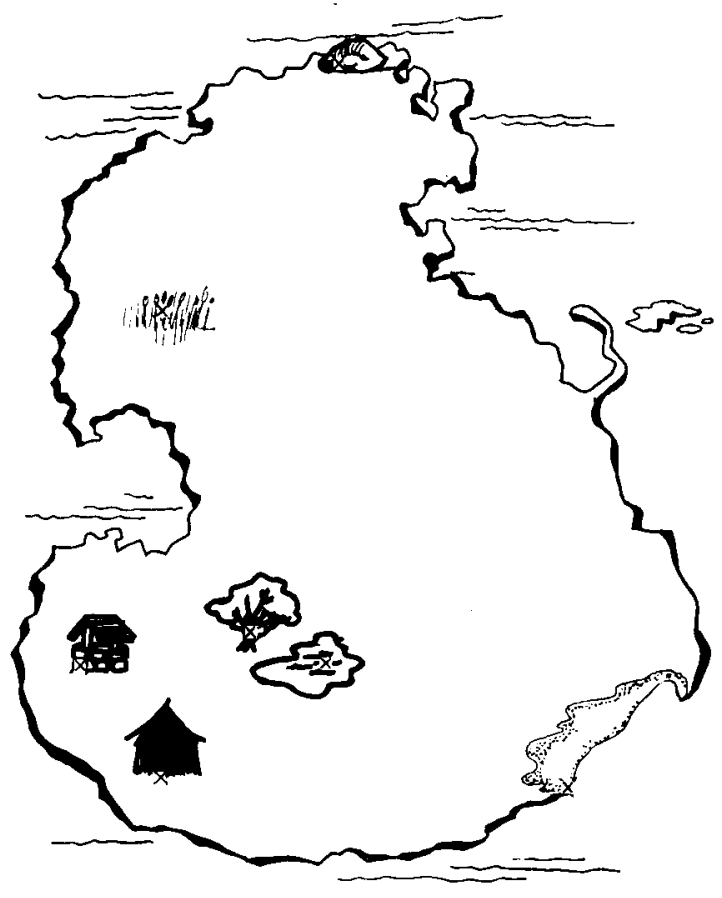

Figure 1. The map imaged and scanned in Experiment 1. 
scan times were discussed at length. The seven items on the map were positioned such that there were 21 distinct distances between them.

A tape recording was also prepared, which contained 42 "true" and 42 "false" trials. The true trials were composed by pairing the name of every item on the map with each other name, so that each pair occurred twice, with different orders of the two names. Each name also occurred six times as the first word in a false pair, in which the second word was not the name of an object on the map (e.g., "bench," "cave," "church"). All names had single syllables and were dissimilar in sound. The tape was constructed such that there were $5 \mathrm{sec}$ between words in a pair and $10 \mathrm{sec}$ between trials. A clock started upon presentation of the second word in a pair and was stopped when the subject pressed either of two response keys. Eight practice trials, four true and four false, immediately preceded the test trials; these trials used names of cities of the United States and Europe as stimuli.

Procedure. The experiment used the same procedure as was used by Kosslyn et al. (1978). We began by asking the subjects to memorize the map. They were instructed to study the map, close their eyes and form an image of it, and then to compare the image with the actual map. This procedure was repeated until the subject felt he or she had an accurate image of the map. At that point, the map was removed, and the subject was asked to draw the locations of the seven objects. This drawing was then placed over the actual map, and the subject studied his or her mistakes until the image was correct; the draw-and-study procedure was repeated until the subject drew each location to within $6 \mathrm{~mm}$ of its actual position.

Once the map was memorized, it was removed, and the subject was asked to form an image of it. A location name then was presented, and the subject was to "focus" on this location while keeping the entire map in view in the mental image, and then to wait for a second name. Upon hearing this name, he or she was to "look for" the item on the imaged map. If the item was on the map, he or she was to scan to it; scanning was to be accomplished by imaging a small black speck moving in a straight line as fast as possible to the object. (This procedure was used because we wanted subjects to scan directly to the second object, given that we are using time as a kind of "tape measure" to infer the existence of a spatial representation.) As soon as the dot hit the second object, the subject was to press one button; if the subject "looked" and was unable to "see" the second object, he or she was to press another button. The subjects were asked to respond as quickly as possible while still following the instructions and keeping errors to a minimum.

We told the first experimenter that we expected a U-shaped function, with the longest times representing the very short and long distances: for the short distances, he was told, it would be difficult to discriminate among the objects that were cluttered together, whereas for the long distances more time should be required to scan. The intermediate distances, we claimed, should be fastest because the items were clearly discriminable and not much distance had to be scanned across.

After the experimenter tested 12 subjects, the data were examined. The results indicated that time increased linearly with distance. At this point, one of the authors expressed unhappiness with the data and suggested to the experimenter that perhaps the subjects were not following the instructions, given that the results were not correct. The effects of the Gestalt Laws were tried and true, he pointed out, and the experiment was almost guaranteed to work as expected. So the experimenter was asked to test 8 more subjects. When these data were collected, the experimenter was asked to select the 12 subjects who followed the instructions the highest percentage of the time (the 12 "best" subjects), according to the postsession reports that the experimenter had collected.

After the results from the first experimenter were collected, a new experimenter was recruited. This experimenter was told the "prediction" and also told about the failure of the first experimenter, who-it was implied-may have been doing something wrong. The author expressed great confidence in the new experimenter's ability to conduct the experiment correctly. This experimenter then tested an additional 12 subjects.

\section{Results}

Preliminary analyses revealed that there were no significant differences in the results from the two experimenters, and the results from the 12 "'best" subjects (as selected by the first experimenter) were no different from the others. Thus, we only present here the analyses based on all 30 subjects.

The mean response time for each distance is displayed in Figure 2. Contrary to the expectations of the experimenters, the results were very similar to those reported before: As in the original experiment reported by Kosslyn et al. (1978), there was a large effect of distance on response times $[\mathrm{F}(20,580)=5.94, \mathrm{p}<.0001]$, in which times increased linearly with increasing distance $[F(1,29)=13.04, p<.002]$, as tested by a linear contrast in the ANOVA. The correlation between response time and distance was $(r=.88, p<.0001)$. A quadratic contrast over distance was also computed, which corroborated the lack of a visible curvilinear trend in Figure 2 $[F(1,29)=2.81, p>.09]$. As can be seen in Figure 2, there was no tendency for subjects to take more time to respond with the putatively cluttered items; times increased with distance, contrary to the experimenters' expectations. In addition, the rate of scanning was similar to that found by Kosslyn et al. (1978) $(58 \mathrm{msec} / \mathrm{cm}$ here compared to Kosslyn et al.'s $45 \mathrm{msec} / \mathrm{cm}, \chi^{2}=.08$, df $=1, \mathrm{p}>.25$ ). If anything, the slope here is steeper than the original one, which is opposite what would be expected if experimenter expectations had succeeded in retarding times for the shortest distances.

The overall error rate was very low ( $1.4 \%$ of the trials), and errors did not vary systematically with distance scanned.

Finally, when the experimenters were debriefed, they displayed genuine surprise upon learning of the actual

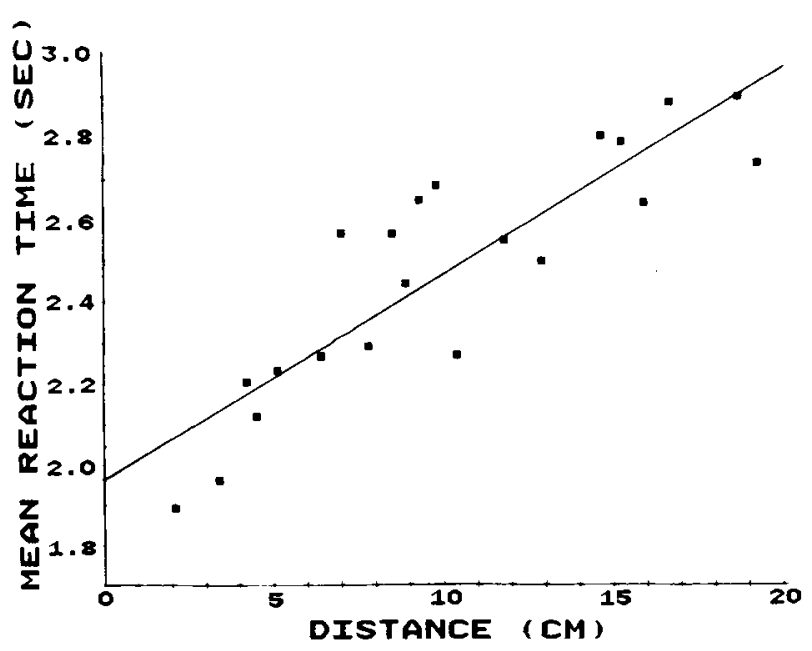

Figure 2. Results from Experiment 1. 
predictions. Neither experimenter expected that times would increase with increasing distance, with times from the cluttered items actually being fastest.

\section{Discussion}

The results nicely replicated the earlier ones: In all cases, time to scan increased with increasing distance. There was no hint of a U-shaped function, as was strongly expected by the experimenters who tested the subjects. These results, then, suggest that the shape of the imagescanning function reflects the operation of underlying mechanisms, and is not due to subjects' responding to cues given to them by the experimenter. The shape of the function is critical, because virtually all theoretical inferences from image-scanning studies have focused on the increased times with distance; the absolute times have not been of much interest in their own right at this point in theorizing.

\section{EXPERIMENT 2}

The results of Experiment 1 suggest that the effects of distance on scan times are not simply due to experimenter expectations. Intons-Peterson (1983) found that the relative overall time to scan images was affected by experimenter expectations, but the slope was unaffected. In this experiment, we test directly the hypothesis that the experimenter's expectations typically affect overall times (the mean) but not the amount of increase with distance scanned (the slope).

Four experimenters asked groups of subjects to scan images of two versions of the map used in the original Kosslyn et al. (1978) experiment; one version was in color, the other in black and white. Two of the experimenters were told that the subjects should in general scan the black-and-white map faster than the colored one (purportedly because the added detail makes the colored one more difficult to keep in mind), and two experimenters were told the opposite prediction (purportedly because the more vivid colored map would be easier to imagine). In addition, one experimenter receiving each of these "predictions" about overall times was also told that we expected slower rates of scanning for the colored map (purportedly because there was more information between the locations on the map than in the black-and-white version), and the other two experimenters were told that we expected slower rates of scanning for the black-and-white map (purportedly because the black-and-white version is less vivid, and the more vivid images are easier to scan across).

Thus, the design of this experiment allows us to compare the effects of experimenter expectation on both the slope and overall mean of the scanning function. In point of fact, the Kosslyn and Shwartz theory (see Kosslyn, 1980) leads us not to expect any effects of color on either parameter.

\section{Method}

This method section has two parts: First we describe the materials and procedure used to test all subjects; this is the within-subjects part of the design. Following this, we describe the training procedure used for each experimenter; this is the between-subjects part of the design.

Subjects. The subjects were 48 adults, some of whom were selected at random from the University of Saskatchewan Psychology Department subject pool, and some of whom were acquaintances of the experimenters. Each subject was tested only once, by a single experimenter, and no subject in this experiment partic ipated in any other experiment reported in this paper.

Materials. Two versions of the map used in Experiment 1 were used in this experiment. The black-and-white map was identical to that used in Experiment 1 except that the four closest objects were not drawn in especially heavy ink (i.e., the map was identical to the one used in the original Kosslyn et al., 1978, experiment). The color map was a copy of the original map that was brightly colored (the interior was yellow-orange, the grass was green, the lake blue, the tree green, the hut red, the well brown, the rock brown, and the beach tan; the surrounding ocean was blue). The spatial layout of the landmarks on the map were identical in the two versions.

The names of the landmarks were paired in all possible combinations. Two lists of these pairs were prepared. Each list contained 42 pairs, formed by each possible pairing of the 7 landmarks in each order (not including pairs in which both members were the same). The pairs in each list were ordered such that the 21 possible pairs were included once in the first and second halves of the list. In addition, each landmark name was used as the first member and the second member of a pair equally often in each half of the list. The two lists differed in the order of the pairs within each half, which was determined randomly within the above constraints. The lists of pairs of landmarks were tape recorded onto two separate tapes (Tape 1 and Tape 2).

Two versions of an additional map were also used in practice trials. This map depicted the locations of four cities in the province of Saskatchewan, which were very familiar to the subjects. One version was a black-and-white outline, and one version was colored. The coloring scheme used for this map was similar to that used for the map of the island. A separate tape recording of the six possible pairs of the four city names was also prepared.

Finally, because we counterbalanced the order of the conditions, we used two sets of written instructions, one for each order.

Procedure. The procedure was divided into two parts: learning the map, and scanning the imaged map.

\section{Map learning}

Half of the subjects began with the colored map, and half began with the black-and-white map. Only one map was learned initially, followed by the image-scanning trials before the other map was learned. If the colored map was learned second, subjects were told to add the color to their memorized map, and to study the new version until they could image the map in full color. If the black-andwhite version was learned second, subjects were told to study the new version and to eliminate the color, imaging only the blackand-white drawing. Otherwise, the map-learning procedure was identical to that used in Experiment 1.

\section{Image scanning}

The image-scanning phase of the experiment was similar to that used in Experiment 1. However, unlike the earlier procedure, there were no "false" trials; all stimuli named pairs of items that were on the map. In this experiment, subjects had only one response key. Upon hearing the first word in a pair, subjects were to focus on the location named, as in Experiment 1, while maintaining an image of the entire map. Subjects then pressed the key, which started the tape recorder and caused the presentation of the second member of the pair. Upon presentation of this word, which started the clock, subjects were to scan to the named location in their image. As in Experiment 1, subjects were asked to imagine a small black speck moving as rapidly as possible in a straight line connecting 
the two locations named in the pair. Subjects pressed the key when the speck reached the second landmark in their image, and the clock stopped. The scanning phase of the experiment was controlled by a microcomputer that turned the tape recorder on and off and recorded response times. The instructions to subjects were read aloud by the experimenter (as was done in Intons-Peterson's experiments).

Each subject received a total of 84 scanning trials for each map, hearing both Tape 1 and Tape 2 . Half of the subjects received the colored map first, and half received the black-and-white map first, and the order of the tapes was counterbalanced within each of these groups.

Immediately prior to the testing in each condition, subjects were shown the practice map (in the same color as the test map) and received the six practice trials. Experimenters interviewed subjects after three of the practice trials to ensure that the subjects understood and followed the instructions.

Experimenter training. The experimenters were three advanced psychology undergraduates and one recent biology graduate at the University of Saskatchewan. The three psychology students performed the experiments as part of a course requirement in a laboratory course in cognitive psychology. The fourth experimenter was a paid research assistant. Each experimenter tested 12 subjects.

Four sets of written predictions, graphs of these predictions, and rationales for the predictions were used to help induce expectations in each experimenter. The graphs of the predictions are presented in Figure 3. Each experimenter also received a detailed outline of the experimental procedure to be used when testing subjects.

Each experimenter was given a prediction about the effect of color of the map on the overall response time and a prediction about the effect of color on the rate of scanning. Two experimenters expected the black-and-white map to lead to slower responses than the colored map, and the other two expected the reverse pattern. One experimenter in each group expected the black-and-white map to be scanned at a slower rate (per unit of distance) than the colored map, and the other expected the reverse pattern.

The experimenters were briefed individually by one of the authors, and the predictions and rationale were discussed at length. Questions were answered and additional experimental support for the predictions was supplied (e.g., the effects on scan times of amount of material to be scanned over in Experiment 1 of Kosslyn et al., 1978; and the effects of complexity on image inspection times, described in Kosslyn, 1980). The experimenters were told that they were completing a subset of a larger experimental design. Because the other experimenters were in the same course, and because all of the experiments were using the same laboratory and equipment,

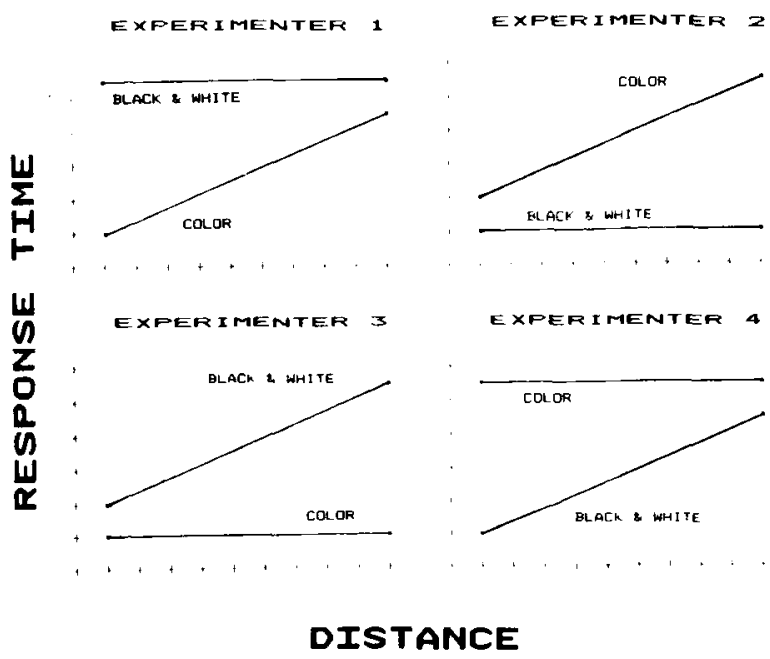

Figure 3. Predictions given to the four experimenters in Experiment 2. we stressed that the experimenters should not discuss any aspect of the experiment. We stressed that strict ignorance of the other parts of the experiment was absolutely necessary to fulfill the independence assumptions of the ANOVA.

\section{Results}

A mean time for each distance scanned was computed for each map and each subject. (Outliers, times more than twice the mean of the other three observations for a given distance, were not included in the mean.) These times were submitted to an ANOVA examining effects of distance, the color of the map, the experimenters' expectations about the overall mean depending on the color of the map, and the experimenters' expectations about the rate of scanning depending on the color of the map.

Figure 4 illustrates the mean response time at each distance for each color of map and for each experimenter. As is evident, scanning times overall again increased with the distance scanned $[\mathrm{F}(20,880)=12.8, \mathrm{p}<.0001]$. The linear trend over distance was highly significant $[\mathrm{F}(1,44)$ $=16.58, \mathrm{p}<.0002]$, and the correlation between overall mean response time and distance was very high $(\mathbf{r}=$ .92). These results, then, replicate the usual imagescanning results reported earlier.

The corresponding panels in Figure 3 illustrate the respective experimenters' expectations about these results. First, consider the effects of experimenter expectation on the rates of scanning the two maps. As is evident in Figure 4, there was no significant effect of experimenter expectation $(F<1)$ for the interaction between slope expectation, color of map, and distance. The expected interaction also was not apparent when the linear trends over distance were compared for the slope expectations associated with different colored maps $[F(1,44)=1.28, p$ $>$.25]. Thus, there were no effects of experimenter expectations on the slopes of the scanning functions.

Now consider the overall means, which should have varied depending on the experimenters' expectations. The mean response time for subjects tested by experimenters who expected the black-and-white map to be scanned faster was $1.376 \mathrm{sec}$ and $1.408 \mathrm{sec}$ for the black-and-white map and colored map, respectively; the mean time for the experimenters who expected the reverse pattern was $1.106 \mathrm{sec}$ and $1.080 \mathrm{sec}$ for the black-and-white map and colored map, respectively. Although there was a very small tendency (no more than $32 \mathrm{msec}$ in either direction) for the differences to be as the experimenters expected, this difference did not approach statistical significance ( $F$ $<1$ ). This was a surprising result to us, as we fully expected to replicate Intons-Peterson's finding.

In addition to the effect of distance, only three other results approached significance in the ANOVA. First, generally slower response times were observed for subjects tested by experimenters who expected subjects to require generally more time to scan the color map $[\mathrm{F}(1,44)$ $=3.73, \mathrm{p}<.06]$. Second, these subjects also had : different pattern of means across distance than did the subjects tested by the other two experimenters $[F(20,880)=$ $1.89, \mathrm{p}<.011]$. However, the two groups did not diffel 

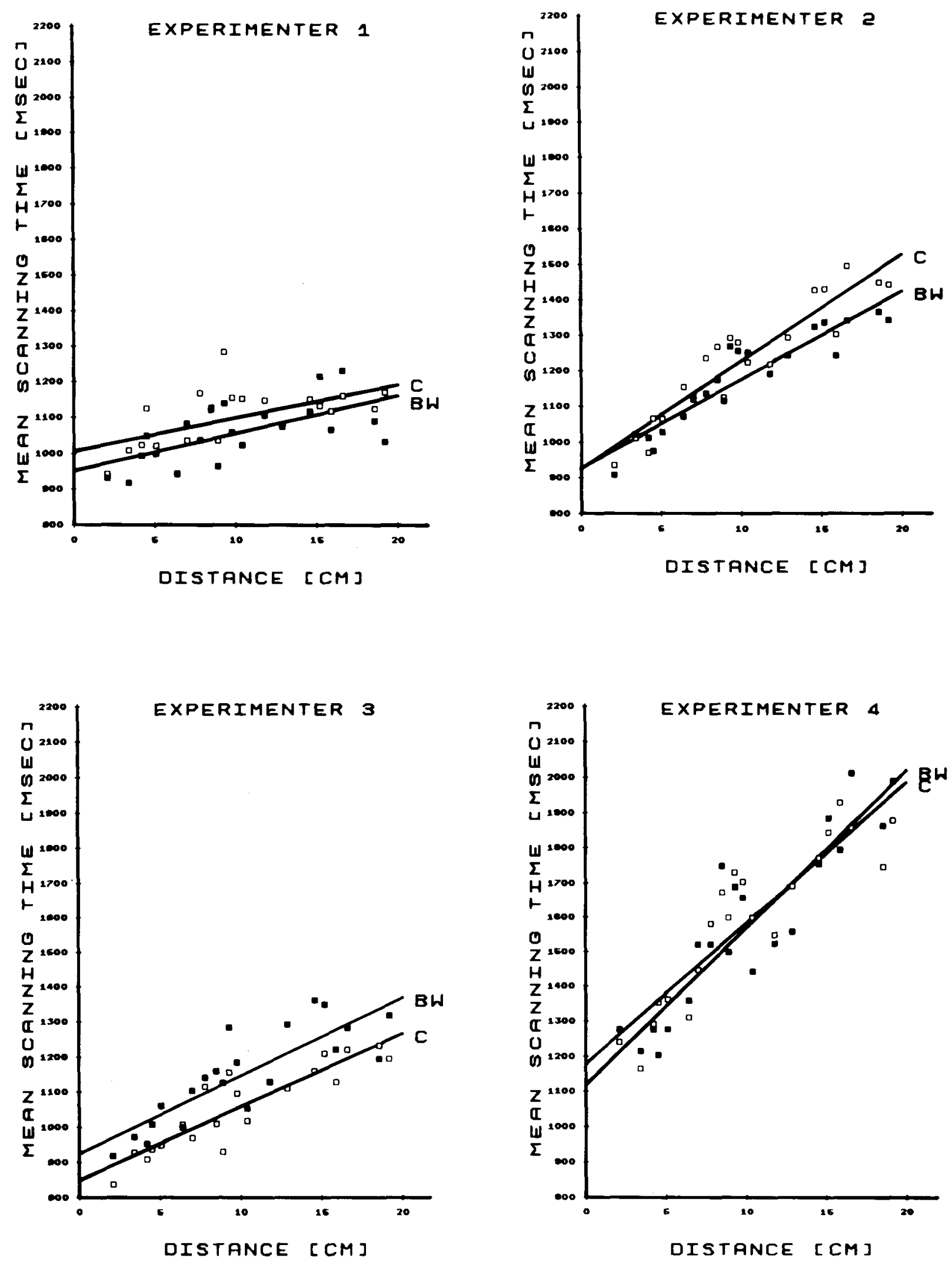

Figure 4. The effects of experimenter expectations, map color, and distance on response time in Experiment 2. The experimenter's expectations for the results are illustrated in the corresponding panels of Figure 3 . The filled squares represent the results for the black and white map; the unfilled squares are for the color map. 
when the linear trends across distance were compared $[F(1,44)=2.36, p>.13]$. The observed differences between the groups could not be due to experimenter expectation, because these expectations hinged on the effects of map color, and the above differences did not depend on map color.

The remaining significant result revealed by the ANOVA was a four-way interaction between experimenter expectation about slopes, experimenter expectation about overall mean, color of map, and distance $[F(20,880)=1.60, p<.05]$ (see Figure 4). However, this interaction did not reach significance when the linear trends over distance were compared $(F<1)$. This pattern of results is difficult to interpret and may reflect nothing more than individual differences. Because it does not directly bear on the hypotheses being examined here, which concern overall times and slopes (linear trends), this interaction will not be discussed further.

Experimenter debriefing. The experimenters were interviewed individually after they had finished testing the subjects. All four experimenters expressed surprise when they learned that we had not in fact expected any effects of color either on the overall scanning times or the rate of scan. All professed to believe the predictions throughout the testing phase. However, one of the experimenters expressed genuine doubt that the results would show any significant distance effects at all. This doubt seemed to be based on his informal observation that responses for some subjects did not seem related to distance. Nevertheless, his subjects showed the usual distance effect (as is illustrated in the lower right hand panel of Figure 4).

\section{Discussion}

Most importantly, we found that the experimenters' expectations about the relative rates of scanning did not affect the results; there were no effects on the slope of the scanning function. Thus, our six experimenters have failed to influence the effects of distance scanned on scan time (in Experiments 1 and 2). This was true even though our experiments were designed to maximize the possibility that we would find experimenter effects: The experimenters read the instructions aloud to subjects, and they were present throughout the map-learning procedure and during the actual scanning trials. Furthermore, the series of practice trials was interrupted midway to allow the experimenter to question the subjects about how they were performing the task, which was designed to provide an additional opportunity for experimenters to influence the subjects' behavior.

It is noteworthy that Intons-Peterson's and her experimenters' expectations also failed to affect subjects scanning behavior. If, as they expected, subjects scanned at different rates depending on the experimenters' beliefs, then the slopes of the scanning functions (which estimate the rate of scanning) should have varied depending on these beliefs, but they did not. Thus, our results replicate Intons-Peterson's finding that experimenters' expectations do not influence the rate of image scanning.

\section{EXPERIMENT 3}

In Intons-Peterson's scanning experiment, the experimenters had different expectations about performance in an imagery condition and performance in a perceptual control condition. In contrast, in our Experiment 2, experimenters had expectations about performance across two imagery conditions. Perhaps experimenters' expectations can influence subjects' behavior more easily or strongly across experimental conditions that are perceived to be clearly different by the experimenters and/or the subjects.

In this experiment, we approximate more closely the conditions in Intons-Peterson's scanning experiment by examining scanning in an imagery condition and scanning in a perceptual control condition. Otherwise, the experiment was identical to Experiment 2. Four experimenters were given different expectations about the rate of scanning (slope) and about the overall mean in the usual imagescanning experiment (Kosslyn et al., 1978) and in a perceptual control condition. Two of the experimenters expected scanning in the imagery condition to yield shallower slopes than scanning in the perceptual condition (purportedly because imaginal scans are "pure" shifts of attention that do not require eye movements, whereas eye movements are required in the perceptual condition). The other two experimenters were told that perceptual scanning would be faster, per unit distance, than imaginal scanning (purportedly because saccadic eye movements have a velocity that is proportional to the distance of the movement, and thus would not show much of a distance effect, in contrast with the imaginal scans in which continuous attentional shifts would be required). In addition, one experimenter in each of the above pairs was also told that perceptual scanning would result in slower response times overall compared with imaginal scanning (purportedly because saccadic eye movements take a long time to initiate). The other experimenter in each pair was told that perceptual scanning would result in faster times overall (purportedly because the imagery condition involves holding an image in working memory, and memory loads tend to slow down performance in general).

As in Experiment 2, the design of the experiment allows us to compare effects of experimenters' expectations on the slope and overall mean of scanning results. In addition, this experiment involves expectations conditional on scanning a memory image versus scanning a perceptual image, which replicates the conditions of IntonsPeterson's scanning experiment.

\section{Method}

The method was almost identical to that used in Experiment 2. The departures from the method outlined in Experiment 2 are described below.

Subjects. The subjects were 48 adults recruited by posting signs at the University of Waterloo. Each subject was tested by a single experimenter and was paid $\$ 4.00$. No subject participated in any other experiment reported in this paper. 
Materials. Only the map used in the black-and-white condition of Experiment 2 was used in this experiment.

Procedure. The procedure was identical to that used in Experiment 2 except for one difference: Subjects scanned either an imaged map or a map physically present before them.

Experimenter training. Experimenters were briefed individually and given "predictions" about the outcome of the experiment. These "predictions" were given verbally by one of the authors and also in written form along with rationales supported by experimental evidence (e.g., the fact that the speed of saccadic eye movements is proportional with distance) and accompanied by a graph displaying the expected pattern of results. Each experimenter was trained by a research assistant and tested one of the authors before testing actual subjects. The expectations held by each experimenter can be seen in Figure 3 by replacing the black-and-white condition by the imagery condition and the color condition by the perceptual condition.

\section{Results}

The data were analyzed as in Experiment 2. Figure 5 illustrates the mean response time for each distance, condition (imagery/perceptual), and experimenter. Scanning time again increased with the distance scanned $[\mathrm{F}(20,880)$ $=23.19, \mathrm{p}<.0001]$, with a strong linear component $[\mathrm{F}(1,44)=37.54, \mathrm{p}<.0001]$. The correlation between mean scanning time and distance was $\mathrm{r}=.84$ in the imagery condition and $r=.88$ in the perceptual condition ( $\mathrm{p}<.0001$ in both cases). Again, we replicated the usual image-scanning results.

As in Experiment 2, experimenters' expectations about the relative slopes of the scanning results depending on condition did not influence the results $(F<1)$, and there was no evidence that expectations affected the overall mean depending on condition ( $F<1)$. Again, we replicated the important finding that the slope of the scanning results is not influenced by the expectations of the experimenters. As in Intons-Peterson's results, scanning across an image resulted in slower times overall compared with scanning across a physical map $[\mathrm{F}(1,44)=14.07$, $\mathrm{p}$ $<.006]$, and there was no difference in the slopes of the scanning results depending on the type of map $(\mathrm{F}<1)$.

Subjects tested by experimenters who expected the imagery condition to yield slower times overall compared with the perceptual condition tended to respond faster than subjects tested by the other two experimenters $[F(1,44)=4.11, p<.05]$. Given that these results did not depend on which map the subjects were scanning and that expectations depended on the type of map, these results could not be due to experimenters' expectations. They are probably due to individual differences or to some global experimenter effect (for example, friendlier or more attractive experimenters might induce faster responding in general). Only one other effect was significant in the ANOVA: the interaction between experimenters' expectations about the overall mean, condition, and distance $[\mathrm{F}(20,880)=1.63, \mathrm{p}<.041]$. However, this interaction was not significant when considering the linear trend across distance $[F(1,44)=2.85$, $\mathrm{p}>$.098], and thus it will not be considered further.

\section{Discussion}

As in Experiment 2, we found that the experimenters' expectations about the relative rates of scanning did not affect the results, and neither did their expectations about the relative overall means. These results provide yet another demonstration that experimenter effects are not as robust and influential in this task as has been previously suggested. Indeed, the authors themselves were surprised by the results; we had fully expected to replicate Intons-Peterson's effects on the overall means.

The final experiment has two purposes. First, the results so far have all turned out to be null findings. These null findings, however, have proven very replicable. Although the possibility of measurement error or lack of power usually makes null findings suspect, the fact that we obtain significant distance and mode (perception/imagery) effects suggests that the null effects of experimenters' expectations are real. Such null findings in the context of other positive findings have had a respectable role in science in the past (e.g., the absence of a yes/no slope effect in the Sternberg, 1966, memory-scanning results). In the next experiment we expect null findings only if the experimenter effect theory is correct; if the imagery theory is correct, we expect an interaction.

The second purpose of the following experiment is to test another kind of possible demand characteristic. One could argue that, even though the experimenters in the previous experiments expected scanning speed to vary, the subjects did not. Perhaps it is the subjects' beliefs about what is expected that are important. This leads us to the second kind of counterinterpretation of the scanning results-task demands, which are considered in the following experiment.

\section{EXPERIMENT 4}

This experiment provides more convergent evidence against the experimenter-effects view, and also provides evidence against the task-demand view. According to the task-demand view, the subjects take it as part and parcel of the task itself to pretend that they are scanning across an actual object, and hence wait longer to respond when longer distances would have been scanned, as presumably would have occurred if objects were being scanned perceptually.

Unlike the previous experiments, in this one the subjects are never asked to scan an image. In fact, the word "scan" is never mentioned in the instructions, eliminating one obvious possible source of task demands. Instead, items are presented that should require scanning an image in order to make the necessary judgment. Furthermore, items that should spontaneously require scanning are randomly interspersed among items that should not require scanning, and the distinction between the items is never mentioned or alluded to in the instructions. Thus, if we find different behavior for the two kinds of items, 

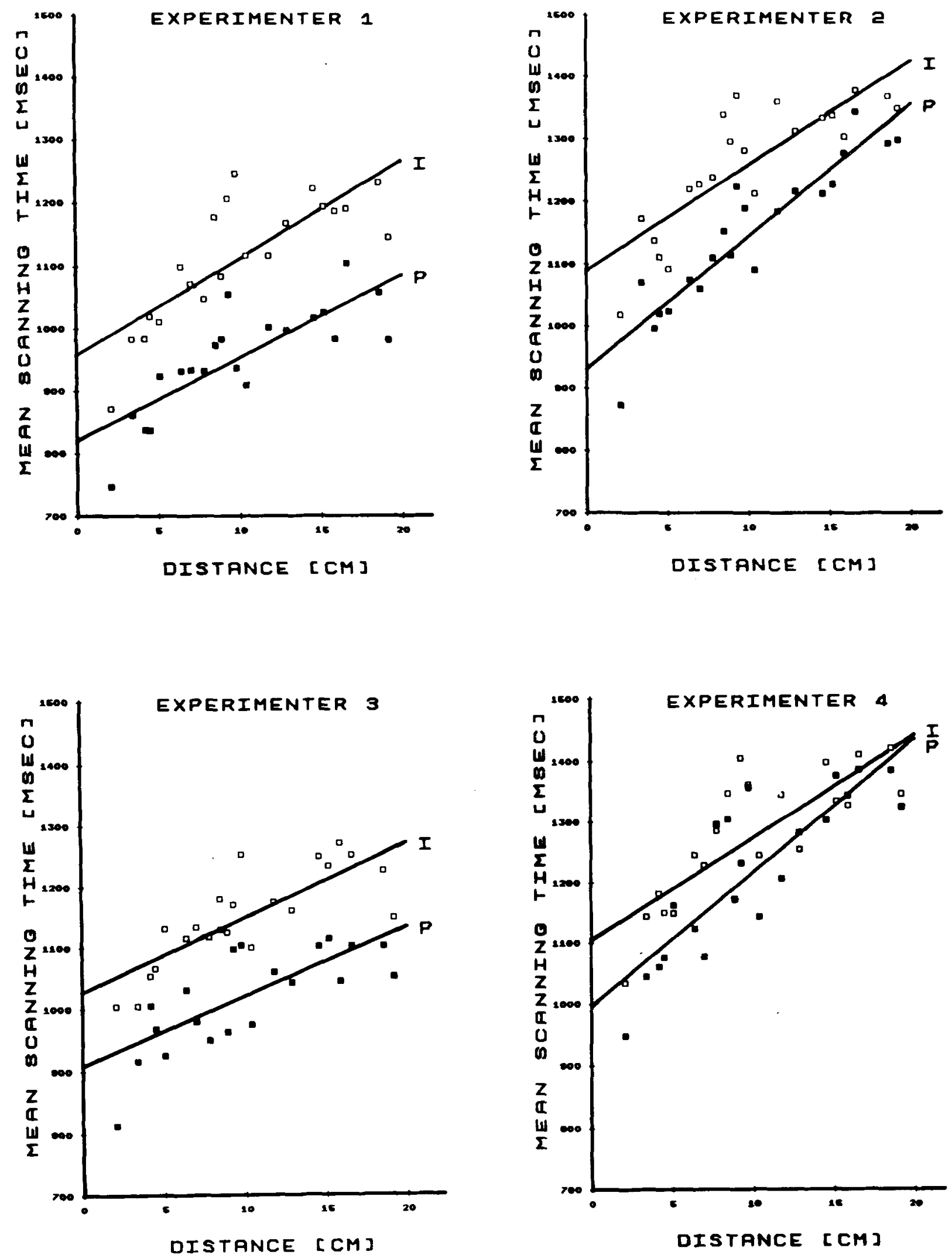

Figure 5. The effects of experimenter expectations, mode (perceptual/imagery), and distance on response time in Experiment 3 . The filled squares represent the results in the perceptual condition; the unfilled squares are for the imagery condition. 
then we have evidence against the notion that the scanning results are due to properties of the instructions themselves.

In this experiment, we asked our subjects to form an image of an object and to focus on one end or the other. We reasoned that if subjects need an image to decide whether an object has a named property, then they will find it easier to use the image already formed instead of generating a new image from scratch (as was found by Kosslyn, 1980, chapter 8). And if they begin by being focused on the inappropriate end of the imaged object, then they will have to scan to the other end, which requires time, before being able to "see" the probed property. The logic of this prediction rests on two assumptions. First, in order to classify parts of an imaged object, one must have them "in focus"; this assumption is justified by previous results showing that resolution decreases with increasing distance from the point of focus in an image, making it increasingly difficult to discern variations in shape (see Kosslyn, 1980, chapter 7). Second, imagery will be required in order to make certain kinds of classifications. This assumption is justified in part by the findings of Eddy and Glass (1981), Kosslyn (1980, chapter 9), Kosslyn and Jolicoeur (1980), and Kosslyn, Murphy, Bemesderfer, and Feinstein (1977), which demonstrate that imagery is used spontaneously to remember some kinds of properties about objects.

Instead of finding scanning effects only for the items that should require imagery, we expect to find scanning for all items if the instructions somehow define a "scanning task demand." And if task demands are all that is responsible for scanning effects and the instructions do not define a scanning task demand, then we should not find scanning effects for any of the items.

\section{Method}

There were two stages to this experiment: the selection of the items and then the testing of subjects in the experiment proper.

Materials. Forty items ( 20 true and 20 false) were selected from a large pool of brief statements that asserted that an object or animal had a specific visible property (e.g., "A stork has a large bill"); all properties consisted of an adjective-noun pair. These items had been rated by a separate group of 20 Harvard undergraduates as to the degree to which each statement required consulting a mental image to decide whether the statement was true or false (see Kosslyn \& Jolicoeur, 1980, for more details of the rating procedure).

All the items selected were elongated either horizontally or vertically when viewed in canonical orientation, the property was always located at one extreme end of the object, and the mean imageryuse rating was either very high or very low (for both true and false statements). For example, "true" high imagery items included "goat-curved horns," "pineapple-pointed leaves," "donkey-long ears," "turtle-short tail," and "bee-black head"; low imagery items included "chimp-two eyes," "rooster-red crest," "submarine-metal propeller," "fencer-face mask," and "elephant-long trunk."

Subjects. Twenty Harvard undergraduates volunteered to be paid subjects. None had participated in the imagery-use ratings task.

Procedure. The subject sat with his or her chin resting on a chin rest and looked at two rectangles, which were mounted at eye level. The rectangles were $24.1 \times 7.6 \mathrm{~cm}$ and were located $91.4 \mathrm{~cm}$ from the chin rest. One rectangle was horizontal; the other was vertical. One end was marked with the letter A, the other with B. A tape recording was prepared in which subjects heard trials of the form, "bee, left. . .A. . .dark head."' Upon hearing the name of the object and its direction, the subject was to "project" an image of the item into the vertical or horizontal rectangle, as appropriate for the shape of the object, facing in the specified direction. The image was to take up the space within the rectangle without exceeding its borders. The letter "A" or "B"' was stated $5 \mathrm{sec}$ later. This cue also turned off the tape recorder. Upon hearing the cue, A or B, the subject was to shut his or her eyes and "mentally stare" at the end of the object corresponding to the end of the rectangle with that letter. As soon as the object was correctly imaged and focused upon, the subject was to press the button, later to be used to indicate "true" properties, which restarted the tape recorder. The name of a possible property was presented $1 \mathrm{sec}$ later. The subject was to decide whether the property in fact was appropriate for the object. Subjects indicated a "true" response by pressing one button, or a "false" response by pressing the other; subjects were to respond as quickly as possible while keeping errors to a minimum. The response stopped the clock, and a new trial began $5 \mathrm{sec}$ later. Half of the items were associated with the A cue, and half with the B cue. Further, half of the items were paired with the cue "left," and half were paired with the cue "right." Two tape recordings were prepared, differing only in which direction was paired with which item, allowing us to counterbalance the direction in which a given item was scanned. Finally, two sets of rectangles were prepared in order to cue the subjects how to focus on their images. The two sets differed only in which letter, A or $B$, was on which end of the rectangle.

Subjects were explicitly told that, although we wanted them to prepare by forming the image and focusing as described, it was not necessary that they use the image. We simply wanted them to make their decisions as quickly as possible, regardless of how they did so.

The experimenter sat behind the subject and recorded both the reaction times and the decisions made. The actual test trials were preceded by eight practice trials, in which the experimenter ensured that subjects prepared for each trial in the correct way but stressed again that the image need not actually be used in reaching a decision. Practice items were drawn from the same pool used to select the test items, but were not rated as clearly high- or low-imagery items. Finally, half of the subjects received one tape, and half the other; each set of rectangles was used equally often with each tape. Thus, each item occurred equally often on the end of the object being focused upon and on the opposite end when the object was facing left and right.

\section{Results}

All analyses were performed on the data from the "true" trials, in which subjects would presumably have had the opportunity to "see" properties on the objects. Mean response times were computed for the high- and low-imagery items when the subject was focused on the end of the object where the property was or on the opposite end. Means were computed after discarding outliers, which were defined as times exceeding twice the value of the mean in a given condition for a given subject; only one score per condition could be so defined. The mean of these means is shown in Figure 6. As is evident, more time was required when subjects focused on the opposite end of the object for high-imagery items, but the same amount of time was required for both focus locations for the low-imagery items. This interaction was only marginally significant in an ANOVA, however $[\mathrm{F}(1,14)=$ $3.61, \mathrm{p}<.08$ ], which is not surprising given the shape of the interaction we expected. However, a contrast test- 
ing the predicted pattern of interaction was highly significant $[F(1,14)=14.92, p<.002]$. Further, in separate analyses of the high- and low-imagery data we found that point of focus affected the high-imagery data $[F(1,14)=$ $7.47, \mathrm{p}<.02$ ], but not the low-imagery data $(\mathrm{F}<1)$. When the two types of items were pooled, more time in general was required for the items not being focused upon $[F(1,14)=5.56, p<.035]$. Finally, as is clearly evident in the figure, high-imagery items generally required more time than low-imagery ones $[F(1,14)=11.02$, $p$ $<.006]$, as is usually the case in these sorts of experiments (e.g., see Kosslyn, 1976; Kosslyn, 1980, chapter 9; Kosslyn et al., 1977). No other effects or interactions were significant in these analyses.

Finally, the error rates were as follows: for focus and nonfocus conditions, $12.5 \%$ and $11.2 \%$ for high-imagery items, and $5.0 \%$ and $2.5 \%$ for low-imagery items. Although the "false" items were not clearly localized on the objects, they were matched for imagery-rating value. High-imagery false items invoked $22.5 \%$ errors, and lowimagery false items invoked $12.5 \%$ errors.

\section{Discussion}

The distance from a focus point to a queried property affected response time only for the high-imagery items. This result was exactly as we would expect if subjects used the image for these items, and scanned to the location where the property should be located. There results are damaging to the task-demands argument for two reasons: First, scanning an image was never mentioned in the instructions, which eliminates the most obvious possible task demand from the usual image-scanning paradigm. Second, the task was identical for all items, and thus the task demands also were identical for all items. If image-scanning results are due to task demands, then subjects either should

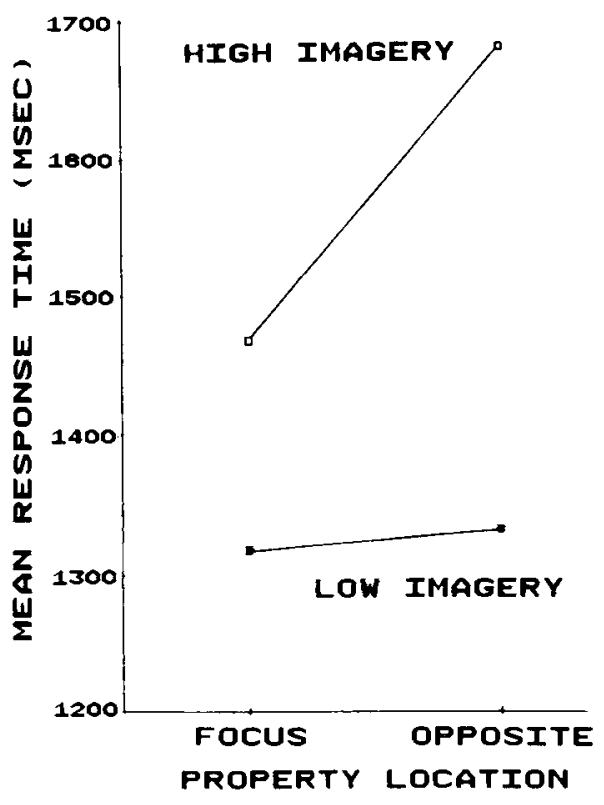

Figure 6. Results from Experiment 4. have pretended to scan for all the items or for none. Therefore, the fact that subjects scanned only the high-imagery items contradicts the task-demands view.

The ardent proponent of the task-demands position could claim that there was something about the items that led subjects to believe that they should simulate scanning only for the high-imagery ones; however, it is not clear how this view would account for the fact that subjects who initially rated the imagery use of the items were able to introspect correctly about which items would later require scanning in this experiment (scanning was never mentioned in the ratings task). The imagery-use judgment was not an imagery analog of a real-world scanning task or the like. Also, it is impossible to disprove a task-demand argument based on the assumption that task demands necessarily had to be at the root of the effects, and thus we have yet to discover what the factors are that produce it (see Pylyshyn, 1981). Unless the argument specifies potentially testable factors or principles, it is useless as a scientific theory. It is true that high-imagery items tend to be less frequently considered, less strongly associated, and less well learned (see Kosslyn, 1980; Kosslyn \& Jolicoeur, 1980; Kosslyn et al., 1977), but it is entirely unclear how these factors could produce the observed results other than by influencing when images are scanned.

\section{GENERAL DISCUSSION}

The results presented in this paper converge in demonstrating that demand characteristics are not responsible for increases in time to scan greater distances across mental images. In the first experiment, we showed that even when experimenters are led to expect the wrong relationship between response time and distance scanned, subjects still produce the correct one. Furthermore, in the second and third experiments, we showed that the experimenters' expectations about the relative rate of scanning two different maps, imaged or perceived, did not affect the actual scanning rates. These results are consistent with all previous results: It has never been found that experimenter effects per se affect actual scanning rates. The strong similarity between the earlier image-scanning findings and those found here should put to rest claims that the effect of distance on time to scan is due to experimenter expectancy effects.

The results from the fourth experiment are inconsistent with both demand characteristics arguments. If experimenter effects were responsible for these findings, they would somehow have to operate selectively to cue the subject to pretend to scan only certain items. However, the experimenter had no opportunity to give such cues, being out of sight, silently recording responses while the experiment was being conducted. In addition, because the task was not defined in terms of scanning per se, it is not clear how the instructions could possibly have been interpreted as requiring selective simulated scanning. Further, the subject was never told that there were two different sorts of items intermixed, and no subject reported even 
a hint of suspicion about the actual purpose of the experiment (most thought it was a memory test).

The present results converge nicely with those of Finke and Pinker (1982, 1983) and Reed, Hock, and Lockhead (1983), which is quite striking, given the dissimilarities in the methodologies. Finke and Pinker showed subjects a field of random dots, followed by an arrow; subjects were to decide whether or not the arrow would point at a dot if it were superimposed over the previously-shown field of dots. The subjects were not told to scan images, but were expected to do so on the basis of theory. The time to decide whether the arrow would point at a dot not only increased linearly with the distance to a dot, but the rate of increase was almost identical to that found in image-scanning studies. This result was replicated by Pinker, Choate, and Finke (1984), who had subjects form their images from long-term memory. Again, these results are very difficult to explain by appeal to the task-demands argument. In addition, our results dovetail nicely with those of Reed et al. (1983), who asked subjects to scan images of diagonal lines, spirals, and mazes, or to estimate scan times without actually performing the scanning task. They found that subjects were unable to predict the effects of different scan paths on actual scan times, which contradicts the notion that the instructions induce subjects to produce the results by drawing on "tacit knowledge" or expectations of how scanning works. In addition, they found that response times were not predicted by the subjects' estimates of the distance to be scanned, as would be expected if task demands led the subjects to respond more slowly when they should have scanned longer distances. Reed et al. concluded that the most parsimonious explanation is that subjects really did scan spatial images in the scanning task.

The present results and those of Finke and Pinker (1982, 1983), Intons-Peterson (1983), Mitchell and Richman (1980), Reed et al. (1983), and Richman et al. (1979) may leave the reader somewhat confused: In some cases researchers find effects of demand characteristics, and in other cases they do not. However, there seems to be one systematic result: Demand characteristics do not affect the slope of scanning functions. Intons-Peterson demonstrated that experimenter effects could affect the intercepts, but neither she nor we found effects on the slopes.

However, in experiments in which subjects' estimates of task performance, and not actual performance, are obtained, demand characteristics can affect the slopes (Kosslyn \& Folk, in Kosslyn, 1983; Kosslyn et al., 1979; Mitchell \& Richman, 1980; Richman et al., 1979). It is informative, then, that these estimates do not seem to be used in actually performing the task. For example, Reed et al. (1983) found that subjects' estimates of the intercepts of the scan functions were affected by the shape of a scan path, but their estimates of the slope were noteven though differences in the scan path had large effects on the slope of the actual function. In addition, when Richman et al. (1979) asked subjects to estimate the effects of distance on the time to scan, they obtained estimates of the slope that were almost 10 times steeper than the slopes actually obtained in scanning experiments.

Why did we persistently fail to replicate the IntonsPeterson results on the intercepts of scanning functions? There are many opportunities for experimenter effects to enter into a procedure, and many of them have been eliminated in our experiments. For example, we ask all subjects to keep their response fingers on the keys at all times. If the experimenter were lax about such instructions for some groups, this would produce a main effect, because more time is required to respond if fingers are not resting on the keys. Or perhaps the experimenters in the different groups were themselves influenced by the author, with the relative enthusiasm of the author resulting in more enthusiastic experimenters, and more enthusiastic experimenters inducing subjects to respond more quickly (as was suggested by Intons-Peterson). These experimenter effects seem quite subtle and unlikely to have affected the earlier image-scanning results reported in the literature, given that the same procedures were used in previous experiments as we used in the experiments in this study.

The main issue we addressed in this paper is whether experimenter expectancy effects and/or task demands are sufficient to account for the results of image-scanning experiments that have been used in theorizing about the nature of mental representation and processes. Based on earlier research on mental scanning, on research on demand characteristics within this domain, and on the present experiments, there is no evidence that demand characteristics provide a sufficient account of the increase in response time with increasing distance. However, it seems likely that instructions could be devised that can affect how subjects actually process information in imagery experiments and that can affect scanning rates per se. After all, imagery gains much of its usefulness as a way of mentally simulating possible real-world events because one can voluntarily vary the speed of scanning and various imagery transformations. More to the point, however, the experiments on image scanning in the literature did not employ such heavy-handed instructions.

Although research on demand characteristics is likely to be valuable to cognitive psychology in general (e.g., by discovering when and how such effects occur), it is unlikely to be very informative with respect to the underlying mechanisms operating in tasks such as mental scanning. At this time, the most parsimonious interpretation of the results on image scanning is that it is the properties of the underlying mental representation that produce the increased times when further distances are scanned.

\section{REFERENCES}

Anderson, J. R., \& Bower, G. H. (1973). Human Associative Memory. Hillsdale, NJ: Erlbaum

EDDY, J. K., \& GLASS, A. L. (1981). Reading and listening to high and low imagery sentences. Journal of Verbal Learning \& Verbal Behavior, 20, 333-345 
FinKe, R. A., \& PINKER, S. (1982). Spontaneous imagery scanning in mental extrapolation. Journal of Experimental Psychology: Human Learning \& Memory, 8, 142-147.

Finke, R. A., \& Pinker, S. (1983). Directional scanning of remembered visual patterns. Journal of Experimental Psychology: Learning, Memory, \& Cognition, 9, 398-410.

InTONS-PETERSON, M. J. (1983). Imagery paradigms: How vulnerable are they to experimenters' expectations? Joumal of Experimental Psychology: Human Perception \& Performance, 9, 394-412.

KossLYN, S. M. (1973). Scanning visual images: Some structural implications. Perception \& Psychophysics, 14, 90-94.

KossLYN, S. M. (1976). Can imagery be distinguished from other forms of representation? Evidence from studies of information retrieval times. Memory \& Cognition, 4, 291-297.

KossLyn, S. M. (1980). lmage and Mind. Cambridge, MA: Harvard University Press.

KossLyn, S. M. (1981). The medium and the message in mental imagery. Psychological Review, 88, 46-66.

KossLYN, S. M. (1983). Ghosts in the Mind's Machine. New York: W. W. Norton.

KossLyn, S. M., BALL, T. M., \& Reiser, B. J. (1978). Visual images preserve metric spatial information: Evidence from studies of image scanning. Joumal of Experimental Psychology: Human Perception \& Performance, 4, 47-60.

KossLYN, S. M., \& JolicoeUR, P. (1980). A theory-based approach to the study of individual differences in mental imagery. In R. E. Snow, P. A. Federico, \& W. E. Montague (Eds.), Aptitude, Learning, and Instruction: Cognitive Processes Analysis of Aptitude, Vol. II. Hillsdale, NJ: Erlbaum.
Kosslyn, S. M., Murphy, G. L., Bemesderfer, M. E., \& Feinstein, K. J. (1977). Category and continuum in mental comparisons. Journal of Experimental Psychology: General, 106, 341-375.

Kosslyn, S. M., Pinker, S., Smith, G. E., \& Shwartz, S. P. (1979). On the demystification of mental imagery. Behavioral \& Brain Sciences, 2, 535-548.

KossLyn, S. M., \& Pomerantz, J. R. (1977). Imagery, propositions, and the focus of internal representations. Cognitive Psychology, 9, 52-76.

Mitchell, D. B., \& Richman, C. L. (1980). Confirmed reservations: Mental travel. Journal of Experimental Psychology: Human Perception \& Performance, 6, 58-66.

PINKER, S. (1980). Mental imagery and the third dimension. Journal of Experimental Psychology: General, 109, 354-371.

Pinker, S., Choate, P. A., \& Finke, R. A. (1984). Mental extrapolation in patterns constructed from memory. Memory \& Cognition, 12, 207-218.

Pylyshyn, Z. W. (1973). What the mind's eye tells the mind's brain. Psychological Bulletin, 80, 1-24.

PylYshyn, Z. W. (1981). The imagery debate: Analogue media versus tacit knowledge. Psychological Review, 87, 16-45.

REed, S. K., Hock, H. S., LockHEAD, G. R. (1983). Tacit knowledge and the effect of pattern configuration on mental scanning. Memory \& Cognition, 11, 137-143.

Richman, C. L., Mitchell, D. B., \& Reznick, J. S. (1979). Mental travel: some reservations. Journal of Experimental Psychology: Human Perception \& Performance, 5, 13-18.

STERNBERG, S. (1966). High-speed scanning in human memory. Science, $153,652-654$.

(Manuscript received July 2, 1984;

revision accepted for publication July 17, 1985.) 